\title{
ANTIBIASED GALAXY FORMATION IN THE PANCAKE SCENARIO
}

\author{
A. Dekel, E. Braun and P. Shapiro \\ Racah Institute of Physics \\ The Hebrew University \\ Jerusalem 91904, Israel
}

We reconsidered the timing problem of the neutrino-dominated cosmological scenario, i.e. the inferred young age for the large-scale structure versus the observationally indicated older age of galaxies and quasars. It can be resolved by 'anti-biasing: of galaxy formation. By applying a variety of alternative prescriptions for galaxy formation to $\mathrm{N}$-body simulations of the neutrino scenario, we find that the clustering of galaxies could be suppressed relative to the neutrinos to an extent that eliminate: the timing problem. It requires that galaxies form preferentially in the flat 'sheets relative to the denser 'filaments' and compact clusters. This could emerge eithe from differences in the efficiency of galaxy formation itself, or due to feedback influ ence from a first generation of objects (e.g. quasars) which suppress the formatio: of incipient galaxies locally. Such an effect could be due to radiation pressure b $L_{\alpha}$ trapping in the ionized region at the vicinity of a quasar. We simulated varioli. examples in order to quantify the requirements from such anti-biasing mechanisms and investigated their physical plausibility. Other aspects of the neutrino cosmology were studied in view of the proposed anti-biasing, concluding that we see no fata flaw in this picture.

Braun, E., Dekel, A. and Shapiro, P. 1987, Astrophys. J. , submitted. 